\title{
Vivencia del ocio entre hombres: los juegos de bocha (São Paulo, Brasil) y bolos tradicionales (Boiro, España) \\ Leisure time experiences among men: bocha's game (São Paulo, Brazil) and traditional skittles game (Boiro, Spain) \\ Luiz Gonçalves-Junior, Denise Aparecida Corrêa, JoséEugenio Rodríguez Fernández \\ *Universidad Federal de São Carlos (Brasil), **Universidad Estadual Paulista (Brasil), ***Universidad de Santiago de Compostela (España)
}

Resumen. La expectativa de vida de brasileños y españoles ha aumentado considerablemente en los últimos años y habitualmente podemos ver grupos de tercera edad organizándose y reuniéndose con el propósito de programar y realizar actividades de ocio, siendo el juego de Bocha uno de los más comunes entre el género masculino en São Paulo (Brasil) y el juego de Bolos en Boiro (España). El objetivo de esta investigación es comprender la vivencia del ocio entre señores practicantes de ambos juegos populares y las similitudes existentes sobre el juego y los participantes de ambos países. Diseñamos un estudio cualitativo/descriptivo, empleando la entrevista individual semiestructurada como herramienta para la obtención de datos. Se realizan 20 entrevistas, con media de edad de 65 años (Brasil) y 73 años (Boiro). Los resultados muestran que el juego de Bocha y de Bolos son los más importantes para los hombres en sus respectivas ciudades, donde es raro encontrar una persona joven o una mujer practicando estas modalidades y en las que destaca su carácter lúdico y no reglamentado en su práctica habitual. Se constatan patrones de comportamiento similares entre los practicantes de Bocha y Bolos, pasatiempo favorito entre personas con edades comprendidas entre los 50 y 75 años y que ambas actividades contribuyen al incremento y diversificación de las actividades de ocio a personas mayores.

Palabras clave. Educación Física, ocio, juegos populares, bocha, bolos, tradiciones.

\begin{abstract}
Life expectancy of Brazilian and Spanish people has increased considerably in recent years and usually there exist many senior citizens groups organizing meetings with the purpose of planning recreational activities, the bocha's game being one of the most common among males in Sao Paulo (Brazil), while traditional skittles game in Boiro (Spain). The objective of this research is to understand the experience of leisure among practitioners of the abovementioned popular games, and to study the similarities existing in these games and the participants involved in them. We used a qualitative/ descriptive study design, employing individual semi-structured interviews as a tool for obtaining data. A total of 20 interviews were conducted on senior citizens with average age of 65 years (Brazil) and 73 years (Boiro). Results show that the bocha's game and traditional skittles game are the most important for men in their respective cities, where it is rare to find a young person or a woman practicing these modalities, which highlights their playful and not regulated nature in its usual practice. Similar standards of behavior are found among players of Bocha and Skittles. Both activities are considered the favorite hobby among people aged between 50 and 75 years, as well as they contribute to the increase and diversification of leisure-time activities in older persons.
\end{abstract}

Keywords. Physical Education, leisure, popular games, bocha, skittles, traditions.

\section{Introducción}

Se vive hoy en una sociedad compuesta, en su gran parte, por ancianos cuya expectativa de vida viene aumentando en los últimos años. Sin embargo, prevalece en esta misma sociedad una valoración extrema del trabajo y de quien lo hace. Con la concepción de utilidad y actividad relacionada al trabajo, es común atribuir al anciano el estereotipo de inutilidad y de pasividad, ideas que se asocian también de forma despectiva al concepto de ocio (Gomes \& Elizalde, 2009).

El avance económico e industrial desencadenado a partir de la Revolución Industrial presentó un aspecto negativo respecto a la posibilidad de vivenciar el ocio por parte de la sociedad, ya que la autonomía para administrar el propio tiempo, posibilitada anteriormente por la realización de actividades esencialmente artesanales en la propia residencia de la persona, termina con la configuración de las industrias como el nuevo sitio de trabajo y con la implantación de la jornada de trabajo fijo. Así, el tiempo de trabajo y el tiempo libre que no tenían límites tan explícitos, pasaron a ser codificados según la visión de la producción y del tiempo mecánico.

En ocasiones se observa la concepción de ocio ligada a la visión utilitaria, que lo considera como reposo y como forma de recuperación de energía después de la jornada de trabajo. Según Magnani (1998), las personas (entrevistadas por él en su investigación) atribuyen más importancia y seriedad a una acción cuando son interrogadas respecto a temas como educación y política, y la desvaloran cuando son interrogadas sobre el tema ocio.

Exaltado en el pasado, el ocio (scholé, en griego) era privilegio de un grupo selecto de individuos, indispensable para una vida feliz, libre, espiritualizada y bella, diferente del trabajo que era visto como deshonor y degradación y aplicado como castigo a los esclavos y a los pobres, principalmente en las sociedades griega y romana. Con la ascensión del

Fecha recepción: 28-02-17. Fecha de aceptación: 24-05-17 José Eugenio Rodríguez Fernández geno.rodriguez@usc.es capitalismo, aquellos valores se invirtieron y se pasó a dar una extrema valoración al trabajo y a ver el ocio como algo pernicioso (Chaú, 1999).

Así, el capitalismo incentiva solamente las actividades de la Industria del Ocio, involucradas con el propósito de controlar tanto el tiempo libre de las personas como la producción y consumo a gran escala de productos ideológicamente estimulados para las personas a través de una extraordinaria publicidad mediática y calificados como indispensables: la televisión táctil de última generación para la práctica y visionado de juegos deportivos; el viaje en paquete de «todo incluido» a modernos complejos turísticos; la adquisición de un novedoso aparato de musculación para la práctica de ejercicios físicos; la ropa con tejido transpirable para atletismo; o los videojuegos o los paseos por un centro comercial, entre otros.

Cada vez más hay palabras en inglés asociadas al nuestro día a día, que nos despierta a pensar que la globalización del mundo involucra especialmente una globalización de la economía y de los productos asociados a las Corporaciones del gran capital, incluyendo, por lo tanto, el campo del ocio, del deporte y de la cultura en general.

No en vano, a pesar de la opresión generada por el capitalismo a las manifestaciones populares del ocio, de por si actividades que no necesitan de gran gasto económico, comprendemos que la práctica social del ocio puede asumir una vertiente crítica, de resistencia y de transformación ante las formas opresivas estructuradas por el capital.

El ocio, en cuanto una manifestación esencialmente humana, puede contribuir para el desarrollo personal y social de las personas en sus comunidades y no debe, conforme advierten Gonçalves-Junior \& Santos (2006), ser comprendido como una práctica social fragmentada en el tiempo (libre o de trabajo); tampoco como posibilidad de realizarse únicamente en espacios estrictamente delimitados (equipamientos específicos de ocio u otros espacios); o cerrado en cuanto a tipología de actividades (lúdicas o no lúdicas); pero si debe ser comprendido de forma prioritaria en cuanto a su intencionalidad.

Prosiguen Gonçalves-Junior \& Santos (2006) en la presentación de esta comprensión afirmando que, no obstante, se observan interferencias de la práctica social/trabajo en la práctica social/ocio y viceversa, así como de otras prácticas sociales. También destacan la necesidad de 
políticas públicas que atiendan a la construcción de equipamientos específicos de ocio.

En cuanto a la actividad, observan que deben ser significativas y llenas de sentido para la persona que la realiza (y que esta no sea obligatoria o coaccionada) implicando, por lo tanto, de forma prioritaria, la intencionalidad atribuida por el ser al ocio (y al trabajo y las demás prácticas sociales), y no desconsiderando el contexto sociopolítico, que involucra opresión (de unos sobre otros) y desigualdades (entre unos y otros). Nos posicionamos de esta forma en una perspectiva de fundamentación fenomenológica-existencial (Merleau-Ponty, 2006), en la cual el ocio no puede ser visto como contrario al pensamiento de una persona, dotando de especial importancia el concepto de intencionalidad.

Sin embargo, la existencia cada vez más grande de grupos de ancianos que se organizan y se reúnen con el propósito de programar y realizar actividades que incluyen arte, deporte y ocio (denominados Grupos de la Tercera Edad), demuestra que además de activos, los señores mayores son capaces de promover su propio ocio, siendo común, entre ellos, la práctica de juegos populares como la Bocha, en la ciudad de São Paulo (Brasil) y de los Bolos, en la ciudad de Boiro (España).

Estos ancianos se reúnen en clubes deportivos, centros sociales, asociaciones, parques públicos o espacios verdes asociados a una taberna para practicar los citados juegos (Rodríguez, Pazos \& Palacios, 2015a). Puntualizamos que, aunque haya la disputa de Bocha y de Bolos en términos deportivos de rendimiento con rígidas delimitaciones de reglas por las Federaciones de los dos países, en esta investigación se enfocan ambos juegos como posibilidades de relación del ocio entre personas mayores.

El objetivo que se persigue en esta investigación es comprender la vivencia del ocio entre hombres practicantes del juego de Bocha en la ciudad de São Paulo (Brasil) y Bolos en la ciudad de Boiro (España), así como las posibles similitudes entre ambos juegos y las características de los practicantes de los dos países.

Concretamente, las hipótesis planteadas son:

1. Es posible identificar patrones de comportamiento similares entre los practicantes de Bocha y Bolos.

2. Se espera encontrar diferencias entre personas de ambos sexos y edades de los participantes en relación a estos dos juegos populares.

3. Los juegos de Bocha y Bolos son los más populares entre la gente mayor, colectivo en el que la oferta de ocio y tiempo libre se reduce considerablemente respecto a otras franjas de edad más cortas.

\section{El juego de Bocha}

En acuerdo con Steiger (1987) el juego de Bocha proviene del período del Imperio Romano y era practicado con el nombre de «Boccie», incluso por los emperadores de la antigua Roma. Durante la expansión de este Imperio fue llevado por los ejércitos de ocupación a todos los pueblos invadidos, convirtiéndose tan popular que era practicado en las plazas públicas y en las calles. Según Steiger (1987), en el siglo XVI el «Boccie» era practicado en Francia, Italia, España, Portugal e Inglaterra, sufriendo numerosas transformaciones desde esa época hasta nuestros días.

En Brasil, el juego de Bocha se introdujo por medio de inmigrantes italianos, que empezaron a llegar a partir del año 1.870, inicialmente a São Paulo, Santa Catarina y Rio Grande do Sul, para posteriormente expandirse por otras provincias del país.

El juego consiste en, básicamente, aproximar las Bochas (bolas más grandes) al Bolín (bola más pequeña), siendo habitual que cada jugador, pareja o trío de jugadores disputen con Bochas de diferentes colores para ver quien consigue aproximarlas más al Bolín, obteniendo en cada jugada un determinado número de puntos. Vence quien, de acuerdo con la perfección de las jugadas, suma un número de puntos superior al resto.

Antiguamente, las Bochas eran de madera, con la superficie compuesta de ranuras que diferenciaban un equipo de otro; actualmente, son de material sintético de aproximadamente $115 \mathrm{~mm}$ de diámetro y 1,15kg de peso. Ya el Bolín tiene 40mm de diámetro.
La Bocha debe ser practicada sobre terreno plano nivelado y limitado por tablados perimétricos de madera u otro material no metálico. La cancha de juego tiene 4 metros de ancho por 26,5 de largo y altura uniforme de 30 centímetros, esto de acuerdo con las reglas oficiales de la Federação Paulista de Bocha e Bolão (2016), aunque puede tener otras dimensiones en función del suelo, del espacio disponible y del interés de los aficionados (deporte u ocio, por ejemplo). En el pasado, el suelo de la cancha era de tierra y hoy, además de éste, hay otros dos tipos: el de hormigón con una fina capa de arena en su superficie y el sintético. El primero es más habitual encontrarlo en los parques públicos y, el segundo, en los clubes deportivos.

\section{El juego de Bolos}

Afirma Ruiz (2000) que existen varias teorías sobre el origen del juego de Bolos, como las que declaran ser de origen romano, celta o germana. Pero resulta imposible por ahora asegurar cuál de ellas es la verdadera, por falta de datos y documentos escritos sobre el tema (Rodríguez, Pazos \& Palacios, 2015b).

Las construcciones y ruinas (puentes, castros, iglesias, termas, etc.) no dejan duda sobre la presencia de pueblos romanos y celtas en toda España, y también en Galicia, donde está ubicada la ciudad de Boiro, en la cual hicimos la parte de esta investigación sobre el juego de Bolos.

Otra información importante sobre la posible llegada del juego de Bolos a España y, en especial, a Galicia, de acuerdo con Fernández (1978) y Braun (1984), se debe a las peregrinaciones realizadas a partir del siglo IX, donde peregrinos de toda Europa acuden a Santiago de Compostela con fines espirituales, trayendo sus costumbres y tradiciones, asentándose muchos de ellos definitivamente en Galicia, estableciéndose nuevos núcleos de población, iglesias, abadías, construcciones variadas y, también, nuevos juegos y divertimentos que eran típicos fuera de Galicia y de España y, de entre ellos, los Bolos (Rodríguez, Pazos \& Palacios, 2014a).

Afirma Ruiz (2000) que el siglo XIX fue la época del esplendor del juego de Bolos en España, tiempo de las boleras populares, donde todos tenían su sitio (los hombres para jugar y las mujeres y niños para mirar). La bolera era el lugar de reunión y juego, generalmente situadas en las plazas de las villas, al lado de las iglesias, pero también ligadas en muchos casos a las tabernas, lugares donde los hombres ponían a prueba las virtudes y defectos propios del género masculino (Rodríguez, Pazos \& Palacios, 2014b).

Son años de desafíos entre paisanos, por merienda o por bebida, en los que se organizaban torneos o concursos, generalmente por las comisiones de los festejos de las villas. No había fiesta patronal que no tuviera su concurso de Bolos, después de misa, donde el toque de campanas anunciaba el comienzo y final de las partidas de Bolos en la bolera o en el campo al lado de la iglesia, reuniendo alrededor de ella jugadores y personas de todas partes (Rodríguez, 2016).

Afirma Rodríguez (2013) que el juego de Bolos fue el más popular de entre los juegos, más vinculado al pueblo y a sus costumbres cotidianas, aunque su práctica no escape también a la nobleza. Destaca también que el citado juego, en Boiro, fue el principal divertimiento de los hombres boirenses hasta la primera mitad del siglo XX.

La Federación Española de Bolos (2014) indica que el campo de juego de Bolos, denominado bolera, debe ser en un terreno horizontal, limpio de hierbas u otros obstáculos que no sean los propios del juego. Dependiendo de la modalidad de juego, el campo puede estar totalmente libre de elementos (sobre todo modalidades de pasabolo) o con tablones para delimitar ciertos espacios, impedir la salida de bolas y Bolos o salvarlos como forma de puntuación (modalidades de derribo).

Pero Rodríguez (2013) afirma que se debe observar que esto no responde totalmente a la realidad en medio popular y mismo deportivo en distintas provincias y ciudades de España, pues existe tal cantidad de variaciones del juego que se podrían hacer innumerables clasificaciones. Podemos encontrarnos con variaciones en los Bolos (número, tamaño, altura, grosor y colocación), la bola (peso, tamaño y forma), en terreno de juego (liso, en ligera pendiente, con obstáculos...), en la forma de 
puntuación, número de jugadores, etc. Detalles todos estos que hacen que la catalogación y definición general de las diferentes modalidades del juego de Bolos sea muy complicada.

\section{Metodología}

\section{Diseño}

Desarrollamos esta investigación mediante un enfoque cualitativo, lo cual, comprendemos, implica en el contacto directo del investigador con las personas y con el ambiente/contexto estudiado, donde los datos colectados son predominantemente descriptivos y sin pretensiones de generalizaciones.

Declara Flick (2015) que la investigación cualitativa estudia el conocimiento y las prácticas de los colaboradores, describiendo las interrelaciones en el contexto concreto de la vivencia de las personas, entre ellas propias y en su relación con el entorno. Este tipo de investigación toma en consideración que los puntos de vista y las prácticas a pie de campo son diferentes como consecuencia de las distintas perspectivas (subjetivas) y de los ambientes sociales relacionados con ellas, donde los puntos de partida para la investigación son las ideas de los acontecimientos sociales, de las cosas o de los hechos que encontramos en el campo social en estudio y la manera en que estas ideas se comunican unas con las otras: compiten, entran en conflicto, son compartidas etc.

Pais (2002) advierte que la expresión método, etimológicamente, significa camino, lo que se hace al iniciar la marcha. De esta forma, el método que nos orientó en esta investigación fue el de observar la realidad «(...) en deambulaciones holgazanas, indicándola de una forma hurona, intentando ver lo que se pasa aun cuando no pasa nada. En ese deambular (...) importa hacer (...) un viaje y no un puerto» (p.35).

\section{Muestra}

La muestra del estudio estuvo formada por 10 hombres practicantes de Bocha en la ciudad de São Paulo (Brasil) (identificados como JB1 hasta JB10, asegurando el anonimato de los participantes) y por 10 hombres practicantes de Bolos en la ciudad de Boiro (España) (identificados como X1 hasta X10, con el mismo propósito de mantener el anonimato de los mismos).

La edad de los participantes brasileños oscila entre los 57 y los 75 años $\mathrm{y}$, la de los participantes españoles, oscila entre los 47 y los 78 años. De entre los señores brasileños entrevistados siete son jubilados, uno todavía trabaja como autónomo realizando servicios generales como albañil y dos, que están alrededor de los 60 años, están desempleados, no consiguen otro empleo y están viviendo con el apoyo de los familiares. De los participantes de Boiro, todos están jubilados, excepto el más joven de los entrevistados, que con 47 años regenta una carnicería del lugar.

\section{Instrumento}

La entrevista individual semiestructurada (Canales, 2006; Tójar, 2006) fue el instrumento de recogida de datos empleado en este estudio, realizadas a partir de cuestiones respecto de la práctica de los citados juegos y de la vivencia del ocio en general. La entrevista es semiestructurada cuando el instrumento de recogida de datos está pensado para obtener informaciones de cuestiones concretas, previamente definidas por el investigador y, al mismo tiempo, permite que se realicen exploraciones no previstas, ofreciendo libertad al entrevistado para disertar sobre el tema o abordar aspectos que sean relevantes sobre lo que piensa.

\section{Procedimiento}

Los investigadores acudieron a los lugares de práctica de ambas ciudades para conectar con el grupo de jugadores de estas modalidades. Bajo criterios como años de práctica, buena memoria, disponibilidad, ganas de colaborar, interés y pasión por el juego, se seleccionaron los 10 jugadores de cada lugar. Posteriormente, en un lugar tranquilo, confortable para el entrevistado, a solas, se realiza la entrevista y se graba en audio y video, bajo consentimiento del entrevistado. Ese mismo día, se realiza la transcripción de la entrevista y se prepara para su posterior análisis.

Hernández (2014) destaca la importancia de la empatía (capacidad para ponerse en lugar del otro para comprenderlo mejor y valorar el mundo que lo rodea desde una clave subjetiva) para posibilitar una interacción entrevistador/entrevistado de auténtica comunicación interpersonal. Mediante la empatía no existen interpretaciones buenas o malas, acertadas o erróneas y, los juicios de valor, así como las claves para a su argumentación e interpretación, las ponen las propias personas de la cultura que se investiga.

Con esta perspectiva, es la empatía que nos permite sentir al otro y, en cierta medida, sentir lo que el otro siente, lo que nos emociona con y como el otro. Según Toro-Arévalo (2005), es la empatía y las emociones por ella que desencadenadas que permiten las actitudes de colaboración, respeto, altruismo y solidaridad.

La empatía no es una habilidad que puede ser ejercida o explotada para ser usada metodológicamente, pero entenderla puede ayudar en el proceso de concientización de la función que desempeña como uno de los elementos constitutivos de la relación intersubjetiva.

La humanización se lleva a cabo en la intersubjetividad, proceso histórico por el cual los hombres y las mujeres constituyen y reconstituyen en la mediación de humanización del mundo (Fiori, 1991). Por lo tanto, cualquier relación que se establece entre dos personas que no se establezca en la intersubjetividad, es en sí deshumanizante, ya que este diálogo entre conciencias no se produce cuando una de las personas involucradas codifica al otro.

La intersubjetividad no es algo espontáneo; se establece a partir de la posibilidad de compartir experiencias que la empatía permite, adicionalmente implica intencionalidad y, por lo tanto, acción. Profundiza Merleau-Ponty (2006, p.18) al respecto al afirmar que el ser, el otro y el mundo están visceralmente encarnados:

Debido a que estamos en el mundo, estamos condenados al significado, y no podemos hacer nada ni decir nada que no adquiere un nombre en la historia [...]. El mundo fenomenológico no es el ser puro, pero el sentido de que se trasluce en la intersección de mis experiencias, y en la intersección de mis experiencias con las de lo otro, el engranaje entre sí; por lo que es inseparable de la subjetividad e intersubjetividad cómo su unidad por la reanudación de mis experiencias pasadas en mi experiencia actual, la experiencia del otro en mí.

\section{Resultados y discusión}

Con respecto a las hipótesis planteadas al inicio de la investigación, los resultados obtenidos mediante las entrevistas realizadas a los participantes de ambos países se reflejan del siguiente modo:

Es posible identificar patrones de comportamiento similares entre los practicantes de Bochay Bolos

Respecto a las actividades de ocio, cuatro señores de São Paulo dicen practicar el juego de Bocha todos los días y, los demás, de una a tres veces a la semana. El Señor JB6, 70 años dice que el juego de Bocha significa:

(...) un ocio muy bueno y saludable, un jugueteo que tenemos, es... sabes, el tiempo pasa y no nos gustaría hacer otra cosa. Si estás trabajando, bien. Si no estás, te quedas en el bar emborrachándote de aguardiente... pero aquí estamos todos divirtiéndonos, tenemos bastantes amigos, sabes. El grupo es amigo y tal (...). Es una cosa que nos deja tranquilos, sabes. Te quedas ahí en vano, te quedas ahí en la cancha, es bueno, es limpio... Es un jugueteo que no tiene lío, no hay nada, jugamos... no hay apuesta (...). Entonces, se hacen apuestas, pero... no me gusta... esto es un juego solamente para el ocio, un juego para la diversión.

Además de la Bocha, algunos señores dicen realizar también otras actividades de ocio como caminar, pescar, cuidar de animales, viajar, jugar billar, dominó, fútbol y malla. Destacamos el Señor JB3, 70 años, que comenta que empezó «(...) jugando Bocha por casualidad, sabes. 
Fui a vivir a Vila Granada y me quedé perdido. Entonces, una persona mellevó a un juego de Bocha, empezó a juguetear hasta... hasta sentir.. una energía pues esto es una energía, sabes». Sin embargo, él dice que también juega:

(...) malla en el Club Deportivo Corinthians (...) y en el parque, el ocio es Bocha. Hoyno había juego en el Corinthians, entonces vine aquí y.... es un ocio que, si llegamos preocupados, nos hace olvidar de todo, sabes. Es muy bueno, es un ocio maravilloso... lo que ya había dicho. Hay los amigos, sabes. Entonces, cuando es sábado y domingo, encontramos los amigos, comemos un... un bocadillo, tomamos aguardiente... lo pasamos así y es eso.

En el caso de Boiro, todos los señores entrevistados afirman que juegan a Bolos de 1 a 3 veces por semana, sobre todo en fin de semana, sábados y domingos por las tardes. El señor X2, de 77 años, comenta que «básicamente los días fuertes son los sábados y los domingos por las tardes»; o el señor X5, de 47 años, que afirma que «...sobre todo el domingo, mañana y tarde. El domingo se aprovecha todo, mañana y tarde, pero más por la tarde».

Comentar en este sentido que a pesar de que 9 de los 10 entrevistados estaban jubilados, su día a día sigue siendo de trabajo, del cultivo en sus propias tierras para autoconsumo, de varias tareas relacionadas con el campo y de ayuda en casa con hijos y nietos. De este modo, no es de extrañar que el tiempo de ocio se reduzca al fin de semana y, aquí, el domingo, pues en muchos casos el sábado es considerado como día de trabajo. Además del fin de semana, festivos, fiestas y romerías no pasan por tener sus partidas de Bolos entre los vecinos. Se pronuncia así el señor X9, de 78 años, «...más o menos días festivos, domingos y en fiestas, que durante la semana no juego, eh?».

Se espera encontrar diferencias entre personas de ambos sexosy edades de los participantes en relación a estos dos juegos populares

En las varias visitas que hicimos a los Parques Municipales de São Paulo, donde los señores brasileños practican la Bocha, fueron raras las veces en que vimos personas más jóvenes (con menos de cuarenta años) o mujeres practicando el juego y, generalmente, cuando allí estaban era para acompañar a uno de los practicantes y no porque fuera habitual su presencia para jugar la Bocha.

Curiosamente lo mismo sucedió en las visitas realizadas a los campos de Bolos en Boiro, donde la presencia mayoritaria era de hombres mayores y la de mujeres y niños (raramente) era para asistir al juego y no para practícalo. En este aspecto, hay que comentar que chicos jóvenes (16-18 años) comienzan a practicar este juego y es habitual encontrarlos en un campo de Bolos en Boiro.

Este hecho de no encontrar mujeres o niños en un campo de Bolos se remonta a los inicios del juego, vinculado casi exclusivamente al mundo masculino, y más aún en las modalidades de derribo, donde la fuerza era la principal exigencia en el juego. De todas formas, la no presencia de la mujer en este juego se debe más al rol tradicional ejercido por la mujer en la sociedad que a sus características físicas (Rodríguez, 2016).

Comentarios como el realizado por X9, de 78 años, atestiguan estos hechos: «A veces las mujeres también acudían a mirar, pero no jugaban a nada. Miraban el juego y nada más. Y alguna vez tocaban la pandereta, cantaban... pero nada más»; o por el X8, de 75 años: «No, las mujeres sólo acudían a mirar y nada más. Si iban a los campos de Bolos era de casualidad, las mujeres a los Bolos iban muy poco».

Losjuegos de Bochay Bolos son los más populares entre lagente mayor, colectivo en el que la oferta de ocio y tiempo libre se reduce considerablemente respecto a otras franjas de edad más cortas

Tres de los entrevistados en São Paulo afirman practicar exclusivamente el juego de Bocha como ocio. Uno de ellos, el Señor JB5, 60 años, declara que solamente practica la Bocha porque es «(...) el único ocio (...).Aquí no tenemos nada. ¿Si no fuera el Parque quésería de nosotros? Entonces encontramos a los amigos, charlamos, jugamos y.... el día pasa (...)». Para él, es «(...) un ocio. Estás con los amigos, charlas, preparas un asado, te confraternizas (...)».
Patlajan (1978) señala que la intensa concentración poblacional, consecuencia del creciente flujo migratorio para las grandes ciudades (como que se observa en la ciudad de São Paulo) verificada especialmente con la instalación de centros industriales, implicó un crecimiento desordenado de estas ciudades, sin la preocupación de realizar un planeamiento urbanístico previo.

El señor JB5 se queja, con razón, de los pocos espacios disponibles en su barrio para el ocio, aunque peor es la situación de los barrios donde la construcción predominante es del tipo de los edificios populares. La ley 7.805/72 (São Paulo, 1972) presentada como propuesta para la ciudad con la pretensión de equilibrar las diferentes «funciones urbanas», siendo una de ellas el ocio, establece que la disponibilidad de espacios e instalaciones de común utilización destinados al ocio debe abarcar todos los espacios del conjunto residencial, correspondiendo, como mínimo, $6 \mathrm{~m}^{2}$ de espacio por participante.

Pero, de acuerdo con Corrêa (2002), lo que se observa en muchos conjuntos habitacionales de la periferia de la ciudad de São Paulo es otra realidad, es decir, la inexistencia de espacios destinados al ocio. Cuando raramente nos encontramos con uno, suelen ser espacios estrictamente de uso infantil, con tres o cuatro elementos lúdicos que forman el área de pasatiempo. En otras ocasiones se encuentra una pista polideportiva, aunque desprovista de materiales o cualquier manutención.

En la ciudad de Boiro, los participantes otorgan al juego de Bolos una importancia muy elevada, ya que para ellos constituye la única forma de ocio disponible, ya no por posibilidad (ya que en el municipio hay oferta deportiva y de ocio para el colectivo de tercera edad, como natación, baile, gimnasia...) sino por libre elección de una actividad que de verdad llena sus pretensiones.

En este caso, vemos como el señor X4 nos comenta que «...se juega y se jugaba a los Bolos para pasar el tiempo, porque antes era lo que había, era donde te entretenías un poco y a lo mejor no ibas a trabajar y te distraías jugando a los Bolos, los sábados, los domingos o por la semana». O también el señor X5, que nos da su visión de la importancia del juego de Bolos en su tiempo de ocio y en su vida: «yo lo que sées que el juego de Bolos desde siempre es importantísimo, el ambiente que se creaba en torno a este juego era muy sano, conocías gente, hacías amigos, amistades... pero era espectacular el respeto a todos los jugadores. Eso no era un juego, era una religión».

El señor X10 nos confirma las escasas posibilidades de ocio de antaño, con lo cual, a la actividad que dedicaban parte de su tiempo, le otorgaban una importancia muy elevada:

«(...) antes no había otro entretenimiento, el juego de Bolos era bastante bueno, que yo sepa, era divertido, así para reunirse, para todo... Era lo que teníamos, no había televisiones ni puñetas, que antes no había cines por aquí tampoco, y estábamos entretenidos jugando a los Bolos (...) Y entonces, ¿qué ibamos a hacer? ¡Jugar a Bolos, no teníamos otro entretenimiento!».

\section{Conclusiones}

En base a los objetivos propuestos para esta investigación y a las hipótesis planteadas en un principio, se establecen las siguientes consideraciones:

- Las personas mayores siguen manteniendo las costumbres de antaño y, entre ellas, la práctica de actividades lúdico-deportivas que realizaban en otros tiempos. A pesar de que actualmente la oferta de ocio y tiempo libre dedicado a personas de la tercera edad se ha incrementado notablemente con el paso de los años (Cuenca-Amigo \& San Salvador, 2016), los hombres siguen siendo fieles a sus prácticas habituales. En este caso, la práctica de Bocha (Brasil) y Bolos (España) sigue siendo el pasatiempo favorito para personas con edades comprendidas entre los 50 y los 75 años.

- Con respecto a las prácticas de ocio tradicionales, percibimos que, en ambas ciudades, tanto la brasileña como la española, la participación de la mujer y de los niños en estos juegos es prácticamente inexistente: en el primer caso debido al rol tradicional asignado a la mujer (MéndezGiménez \& Fernández-Río, 2011; Rodríguez, Pazos \& Palacios, 2016) 
y los niños, que jugaban en espacios diferentes, por dos razones fundamentales: para que no salieran lastimados por las actividades típicas de los adultos (accidentes propios del juego, vocabulario empleado en estos lugares, etc....) y porque realizaban otro tipo de prácticas más acordes con su edad (juegos simbólicos, con implementos más seguros, de poco peso y tamaño reducido...) (Rodríguez, 2016).

- Identificamos que la Bocha y los Bolos son las más importantes actividades de ocio entre estos señores, respectivamente, brasileños y españoles, los cuales acaban por reunirlos también para otras actividades en el grupo de amigos, mejorando su entretenimiento diario y, por consiguiente, su calidad de vida. Señalaban incluso señores de São Paulo que, con un pasatiempo como la Bocha, aunque falten otras opciones de ocio y haya un mayor tiempo disponible a causa de la jubilación, se siente menos aburrido, lo que evita que se entregue a vicios como el consumo excesivo de alcohol.

- Los Parques Públicos Municipales se muestran como los pocos espacios de resistencia al crecimiento avasallador de la ciudad de São Paulo, los cuales aún permiten algún contacto con áreas verdes y libres o canchas para el juego de Bocha, entre otras posibilidades de ocio y que, de alguna forma, permiten el acceso de la población pobre a causa de la gratuidad de su utilización. Sin embargo, aunque el acceso sea posible, no siempre las condiciones materiales y culturales permiten a la población más pobre la vivencia efectiva del ocio, aunque el artículo $6^{\circ}$ (Titulo II, Capítulo II, de la Constitución de la República Federativa del Brasil, de 1988) afirme que sea el ocio un derecho social de todo ciudadano (Brasil, 1988).

- En el caso de Boiro (España) el problema de espacio no constituye un problema, ya que el tamaño de ciudad es totalmente opuesto al de una de las mayores urbes del mundo (São Paulo). Apesar de que con el paso del tiempo el número de campos en esta ciudad española se ha reducido notablemente (se han localizado 86 campos de Bolos desde inicios del siglo XX y, en la actualidad, se encuentran activos 4), el acceso a estos espacios sigue siendo libre y de fácil acceso. En consecuencia, estamos hablando de auténticos centros sociales al aire libre; $y$ decimos sociales porque a través de estos juegos populares y tradicionales se pueden percibir las características de una sociedad que vivió y vive ligado a sus costumbres lúdicas.

\section{Referencias}

Brasil (1988). Constituição da República Federativa do Brasil. Brasilia. Braun, J. (1984). Bolos y cultura. Santander: Artes Gráficas Resma.

Canales, M. (2006). Metodologías de investigación social: introducción a los oficios. Santiago de Chile: LOM.

Chauí, M. (1999). Introdução. En P. Lafargue (Coord.), O direito à preguiça. São Paulo: Hucitec.

Corrêa, D.A. (2002). Domingo no parque: a (sobre)vivência do lazer nos parques públicos municipais da zona leste da cidade de São Paulo (1970-2001). Dissertação (Mestrado em História) - Programa de Estudos Pós-Graduados em História-Pontifícia Universidade Católica de São Paulo.

Cuenca-Amigo, M. \& San Salvador, R. (2016). La importancia del ocio como base para un envejecimiento activo y satisfactorio. Revista de Psicología del Deporte, 25(2), 79-84.

Federação Paulista de Bocha eBolão(2016). Regra oficial da modalidade de Bocha mundial. Recuperado de: http://www.fpbb.com.br/mundial-2016/regra-oficial-bocha-mundial-2016.

Federación Española de Bolos (2014). Reglamentos. Recuperado de: h t t p : / / w w w. f e b o l o s.e s / D a t o s / REGLAMENTO\%20JUEGO\%20BOLO\%20PALMA\%20\%20(28.06.2014).pdf

Fernández, A. (1978). Los bolos en España. Gijón: Baukunión.

Fiori, E.M. (1991). Conscientização e educação. En Textos escolhidos de educação e política, v.2., pp. 65-82. Porto Alegre: L\&PM.

Flick, U.(2015). El diseño de investigación cualitativa. Madrid: Morata.

Gomes, C. \& Elizalde, R. (2009). Trabajo, tiempo libre y ocio en la contemporaneidad. Contradicciones y desafíos. Revista Polis, 22, $1-16$.
Gonçalves-Junior, L. \& Santos, M. (2006). Brincando no jardim: processos educativos de uma prática social de lazer. Anais do VI Educere-Congresso Nacional de Educação, vol. 6, pp.1902-1915. Curitiba: PUPCPR.

Hernández, R.M. (2014). La in vestigación cualitativa a través de entrevistas: su análisis mediante la teoría fundamentada. Cuestiones Pedagógicas, 23, 187-210.

Magnani, J.G.C. (1998). Festa no pedaço: cultura popular e lazer na cidade. São Paulo: Hucitec.

Méndez-Giménez, A. \& Fernández-Río, F. (2011). Análisis y modificación de los juegos y deportes tradicionales para su adecuada aplicación en el ámbito educativo. Revista Retos, Nuevas tendencias en Educación Física, Deporte y Recreación, 19, 54-58.

Merleau-Ponty, M. (2006). Fenomenologia da percepção. São Paulo: Martins Fontes.

Pais, J.M. (2002). Sociologia da vida quotidiana: teorias, métodos e estudos de caso. Lisboa: Imprensa de Ciências Sociais.

Patlajan, T. (1978). Urbanização e lazer. Cadernos de Lazer (3). São Paulo: Brasiliense.

Rodríguez, J.E. (2013). O estudo do xogo dos bolos en Boiro: aspectos históricos, estado actual de práctica e perspectivas de futuro. Tesis de Doctorado. Facultad de Ciencias de la Actividad Física y el Deporte. Departamento de Educación Física. Universidade da Coruña.

Rodríguez, J. E. (2016). O xogo dos bolos en Boiro. Tradición, cultura e educación. Noia: Toxosoutos.

Rodríguez, J. E., Pazos, J. M. \& Palacios, J. (2014a). Costumbres lúdicas en España: el juego de bolos en la etapa final del Camino de Santiago. Movimento, Porto Alegre, 20 (4), pp. 1397-1421.

Rodríguez, J.E., Pazos, J.M. \& Palacios, J. (2014b). El juego de bolos de Boiro: la necesidad dereglamentación de una modalidad de pasabolo atípica en España. Revista EmásF, 28, 17-43.

Rodríguez, J.E., Pazos, J.M. \& Palacios, J. (2015a). Evolución histórica del juego de bolos en Boiro. Revista Retos, Nuevas tendencias en Educación Física, Deportey Recreación, 28, 141-146.

Rodríguez, J.E., Pazos, J.M. \& Palacios, J. (2015b). El juego de bolos en España: estudio de revisión. Revista Cultura, Ciencia, Deporte, 10 (30), 177-185.

Rodríguez, J.E., Pazos, J.M. \& Palacios, J. (2016). El rol desempeñado por la mujer en el juego de bolos de Boiro. Movimento, Porto Alegre, 22 (3), 971-984.

Ruiz, J. G. (2000). Estudios de los bolos en Asturias: aspectos histórico-culturales, modalidades, elementosy materiales de juego. Estado actual de su práctica. Tesis de Doctorado. Universidad de Granada.

São Paulo (1972). Coletânea das leis de parcelamento, uso e ocupação do solo. São Paulo: SEMPLA/PMSP.

Steiger, R.N. (1987). O emocionante espetacular esporte da Bocha. Porto Alegre: Sulina.

Tójar, J.C. (2006). Investigación cualitativa: comprender y actuar. Madrid: La Muralla.

Toro-Arévalo, S.A. (2005). Desarrollo humano y motricidad: una aproximación desde la empatia. Revista Consentido, 1 (1), pp.127-137.

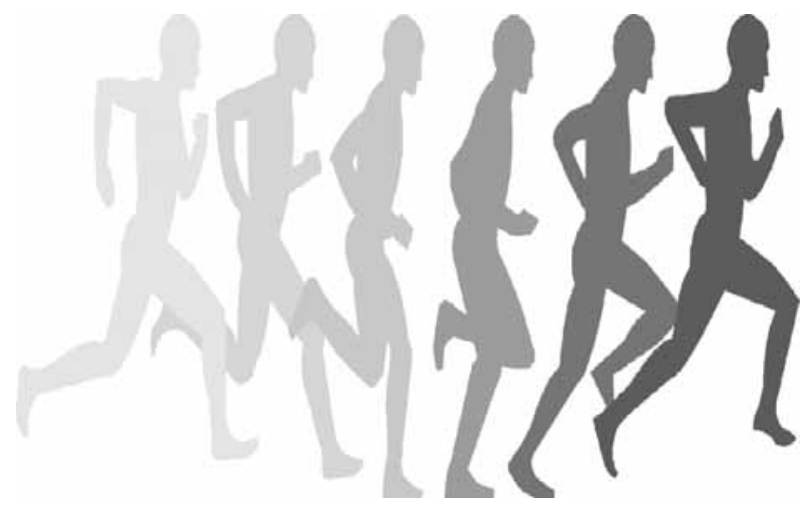

